\title{
Successes and Challenges in Preventing Conduct Problems in Australian Preschool-Aged Children Through the Early Impact (EI) Program
}

\author{
Stephen Larmar \\ Griffith University, Australia \\ Mark R. Dadds \\ University of New South Wales, Australia \\ Ian Shochet \\ Queensland University of Technology, Australia
}

\begin{abstract}
This article reports on the initial effects of the Early Impact (EI) Program, a preventative program that includes home and school components designed to arrest the development of conduct problems in preschool-aged children. Participants included 455 preschool-aged children enrolled across 10 schools. Schools were randomly assigned to either EI or control conditions. All participants were screened at the commencement of the academic year to determine those children considered more at risk for ongoing conduct problems. Following screening $n=66$ children were identified in the intervention group and $n=69$ in the control. The intervention commenced in the second term of the academic year and ran over a 10-week period. Teachers involved in the intervention were highly engaged and satisfied with the program design. Improvements were reported at the school level at post-intervention with mixed evidence of durability of change at 6 -month follow-up. Parent participants were more difficult to engage and reported no changes in the behaviour of children in the home. The study provides initial evidence of the efficacy of the EI intervention as a means of preventing conduct problems in young children at the school level; however, issues associated with parental engagement and home-based change remain to be solved.
\end{abstract}

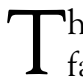
he prevalence of conduct problems in child populations significantly impacts families, educational settings and broader society (Sanders et al., 1999). Recent studies have indicated that child conduct and/or oppositional defiant disorder are part of the developmental pathway of every adult disorder (Kim-Cohen et al., 2003). Conduct problems include behaviours such as aggression, impulsivity, and delinquency. The costs to society in terms of criminal activity, substance abuse and associated psychosocial problems are substantial (Patterson, DeGarmo, \& Knutson, 2000).

Address for correspondence: Stephen Larmar, PhD, School of Human Services, Griffith University, Logan Campus, University Drive, Meadowbrook QLD 4131, Australia.E-mail: s.larmar@griffith.edu.au 
While the literature identifies a vast array of existing treatments to address conduct problems in children, many treatments are often administered too late in the child's trajectory into dysfunction. The literature suggests that early intervention models have potential in the treatment and prevention of conduct problems (August, Realmuto, Hektner, \& Bloomquist, 2001; The Conduct Problems Prevention Research Group, 2002; Webster-Stratton, 1998). In addition, current models of treatment emphasise the significance of influences derived from multiple settings in the individual's world. Such settings include the individual's home and the school environment.

Recent studies have demonstrated the effectiveness of early intervention frameworks. Webster-Stratton (1998) examined the effectiveness of a selected intervention program for preschool-aged children. The program focused on strengthening parenting competencies for parents whose children were enrolled in Head Start Centres. The evaluation involved random assignment of children to either experimental or control conditions. Results identified significant improvement in the behaviour of the intervention group compared with the control (Webster-Stratton, 1998). Walker, Severson, Feil, Stiller, and Golly (1998) evaluated the First Step to Success program. The intervention included universal screening, a school curriculum administered by trained consultants and a parent-training component. Analysis revealed a significant increase in pro-social behaviours in the intervention group and results were sustained at 1-year follow-up. The Fast Track program developed by the Conduct Problems Prevention Research Group (2002) is another program presently being trialled. This indicated intervention consists of parent training and school curriculum implementation by program consultants. Analyses at the 3-year period revealed that children assigned to treatment conditions were less likely to exhibit serious problem behaviours.

These trials show that conduct problems can be targeted for early intervention in young children. However, the early onset of conduct problems in preschool children suggests that earlier screening may be warranted. Existing treatments are designed to target children on their entry into primary school and fail to accommodate preschool children. Further, an intervention that incorporates universal and indicated components in addition to active schoolteacher's engagement in the facilitation of the program would provide greater ecological breadth. Existing frameworks rely on research consultants and fail to empower classroom teachers to facilitate early intervention programs in regular school settings.

The overall aim of the present study was to evaluate the effectiveness of an early intervention and prevention program for preschool children at risk for conduct problems. The specific aims were to examine children and parents' functioning at post-intervention and at 6-month follow-up and to consider the intervention's social validity. It was hypothesised that the intervention would be associated with lower rates of conduct problems at post-intervention and 6-month follow-up, as measured by parent diagnostic interviews and teacher and parent self-reports. Further, it was hypothesised that the intervention would have high social validity.

\section{Method}

\section{Procedure: Recruitment and Screening}

Participants included 455 children with an age range of 4 to 5 years. They were drawn from 10 primary schools in the city of Brisbane, Queensland. Schools were matched in pairs on the basis of socioeconomic status and size and then, within each pair, randomly assigned to be either an experimental or control group. 
Prior to screening, consent forms were sent to parents of children enrolled in the participating schools. Of the initial cohort (635), 455 (89.78\%) questionnaires were completed at pre-intervention. Following recruitment of participants, a screening procedure was used to identify children at risk of conduct problems.

In the first stage of screening, preschool teachers used a class list and a standardised description of externalising behaviours to nominate students considered to be at risk for the development of problem behaviour (Dadds, Spence, Holland, Barrett, $\&$ Laurens, 1997). The standardised description comprised an adaptation of descriptors derived from the text revision of the fourth edition of the Diagnostic and Statistical Manual of Mental Disorders (DSM-IV-TR; American Psychiatric Association, 2001) and the Child Behavior Checklist (CBCL; Achenbach \& Edelbrock, 1991). The list included each child's name and a one-item Likert scale. The scale enabled the teachers to indicate whether each child was often like the child, sometimes like the child, or rarely like the child described in the standardised description. Those children who the teacher perceived to be 'often or sometimes like the child' described were included in the second stage of screening.

A confirmation checklist was used in the second stage of screening to determine participant suitability. Inclusion criteria consisted of age (4 to 6 years), teacher nominations derived from the screening process, parental consent, having no neurological impairment or serious physical illness or developmental disorder (e.g., autism), and absence of attention-deficit/hyperactivity disorder (ADHD), except participants with ADHD who were medicated but continued to present with significant externalising behaviours. The checklist also enabled teachers to comment on other variables including problematic anxiety, and language and learning problems. The final cohort included a balanced gender distribution between the two groups.

\section{The Early Impact Intervention}

The intervention under evaluation was the EI program (Larmar, 2002), a multimodal program comprising a universal school component, a universal home component, and an indicated component.

\section{School Component}

The school component included training teachers and teacher aides in the implementation of strategies of management that could be universally applied. The strategies are delineated in the EI teacher's manual, Encouraging Positive Behaviour in the Classroom (Larmar, 2002a), and encourage democratic class management. Teachers attended a 1-day training workshop to receive instruction in the program implementation, in addition to training in the EI curriculum. An outline of the EI curriculum is provided in the EI program manual, The Early Impact Program: A Program for Encouraging Positive Behaviour in Young Children (Larmar, 2002c). The curriculum includes an intensive phase taught over 10 weeks and an extended phase to consolidate learning over a 6-month period. The curriculum focuses on communication, friendship formation, social problem-solving, self-control, and engaging in pro-social behaviours.

A behaviour consultant was also trained in the implementation of the program. The consultant attended the teacher-training workshop to achieve congruency with the program's goals and philosophy. The consultant worked for one half hour session each week with the indicated students throughout the intensive 
phase, offering supplementary support. Further, the consultant provided additional support to teachers where necessary.

\section{Home Component}

The home component of the EI program consists of parent training. Parenting strategies are presented in the EI parent's manual, Encouraging Positive Behaviour in Young Children (Larmar, 2002b). Parents of children in the intervention group were encouraged to attend parent-training sessions, facilitated over three 120-minute sessions. Parent trainers received 2 days' training in the parenting program. The five trainers held postgraduate qualifications in either education or psychology and had extensive experience in working with at-risk children. The parenting program includes examination of parent's values, beliefs and experiences; parental authority; child development; communication; rules and limits; parent consistency; reinforcing appropriate behaviour and consequences; problem-solving and ownership; assertiveness; managing anger; quality time; and parent preservation. The comparison group received no intervention, but parent participants were informed that their child would receive the intervention in the following school year.

\section{Evaluation Measures}

\section{Family Domain}

All parents were invited to complete baseline self-report measures focused on child behaviour and management practices. These measures were distributed in the eighth-week of the first term of the school year (baseline) and at post-intervention and 6-month follow-up.

Parents completed the parent version of the Strengths and Difficulties Questionnaire (SDQ; Goodman, 1997), which consists of 25 items on five subscales including conduct problems, hyperactivity, emotional symptoms, peer problems, and pro-social behaviour. The SDQ (Parent) served to assess parents' perceptions regarding the extent to which each child exhibited externalising and/or internalising behaviours through the use of a 3-point Likert scale. The SDQ (parent) has moderate to strong internal reliability and validity in Australian preschool samples (Hawes \& Dadds, 2004).

Parents completed an adaptation of the Alabama Parenting Questionnaire (APQ; Shelton, Frick, \& Wooton, 1996) that consists of several subscales identifying positive and negative parenting styles associated with conduct problems in children. Questions from the parenting supervision subscale were revised to reflect parenting practices and child behaviours for preschool children. The APQ consists of 41 items assessing parental function with each item utilising a 5-point Likert scale. The APQ has good internal consistency, validity, and test-retest reliability (Dadds, Maujean, \& Fraser, 2003).

In addition, fourth edition Diagnostic and Statistical Manual of Mental Disorders (DSM-IV; American Psychiatric Association, 1994) diagnostic interviews were conducted at 6-month follow-up using the Diagnostic Interview Schedule for Children, Adolescents and Parents (DISCAP; Holland \& Dadds, 1997). Parents of indicated children, as well as parents of a random selection of non-at-risk children, were interviewed by trained clinical psychologists. The diagnostic interviews served to identify those individuals who met subclinical and full clinical diagnoses at post-intervention. Reliability checks were included as part of the interview process, with a second 
interviewer (listener) engaged in $20 \%$ of the interviews. A reliability standard of not less than $80 \%$ was met for each diagnostic interview and the results were significant at $p<.01$. Correlations obtained for the highest rate of severity on the independent assessments were significant at .97 , indicating adequate reliability. The DISCAP for the diagnosis of DSM-IV disorders in children and adolescents has demonstrated concurrent and discriminant validity (Johnson, Barrett, Dadds, Fox, \& Shortt, 1999).

\section{School Domain}

All teachers in the experimental and control groups completed the teachers' version of the Strengths and Difficulties Questionnaire (Goodman, 1997) for a randomly selected sample of students at baseline, post-intervention and 6-month follow-up. The sample consisted of 5 indicated students, and 5 students representing a normative sample. This randomised process served to reduce potentially stigmatising affects. The SDQ (Teacher) consists of 25 items, and includes five subscales: conduct problems, hyperactivity, emotional symptoms, peer problems, and pro-social behaviour. The SDQ (Teacher) has moderate to strong internal reliability and stability and sound external validity (Goodman, 2001).

Peer ratings were completed in term 1 (baseline) and at post-intervention and 6month follow-up. Children were interviewed using the Peer Nomination Interview Schedule (Larmar \& Dadds, 2002c), which comprises a 12-item measure adapted from an instrument developed by Crick and Grotpeter (1995). The measure includes the following subscales: pro-social behaviour, overt aggression, relational aggression, isolation, peer acceptance, and peer rejection.

\section{Treatment Integrity and Process Measures of Treatment Implementation and Engagement}

To measure teacher consistency in facilitating the program teachers completed the Teacher Self-Report (TSR; Larmar \& Dadds, 2002d), which consists of a 15-item questionnaire. It also measures teacher satisfaction with the program design. The TSR was administered at pre, post, and 6-month follow-up.

To ascertain parent levels of engagement in the home component, parents completed the Parent Self-Report (PSR; Larmar \& Dadds, 2002b) at the conclusion of the intensive phase and at 6-month follow up. The instrument includes five items identifying parents' perceptions of the home component of the program design.

At post-intervention the behaviour consultant completed the Behaviour Consultant Report Form (BCRF; Larmar \& Dadds, 2002a), comprising seven items that ascertain the consultant's level of engagement and the degree to which the consultant utilised remedial strategies.

\section{Results}

ANOVA (SPSS) was used to check equivalence of groups at pretreatment. There were no significant differences as shown in Table 1 . By the 6-month follow-up period 16 children had withdrawn from the trial: 2 children (1\%) from the EI group and $14(6 \%)$ from the control group. The reason for withdrawal was that parents relocated their children to an alternative school. Following screening $n=66$ indicated children were identified in the EI group and $n=69$ in the control. There were no significant differences between the EI and control group for the final cohort of indicated children. 
TABLE 1

Summary of Baseline Characteristics of Participants

\begin{tabular}{|c|c|c|c|c|}
\hline & \multicolumn{2}{|c|}{ Experimental $(n=212)$} & \multicolumn{2}{|c|}{ Control $(n=243)$} \\
\hline & $M$ & $S D$ & $M$ & $S D$ \\
\hline Mean age of children in years & 4.37 & 0.49 & 4.29 & 0.46 \\
\hline No. of siblings & 1.38 & 0.90 & 1.44 & 1.05 \\
\hline Mean age of mother in years & 34.7 & 5.42 & 34.8 & 4.83 \\
\hline \multicolumn{5}{|l|}{ Maternal education } \\
\hline$\% 12$ years or more & 83.2 & & 88.5 & \\
\hline$\% 10$ years or less & 14.42 & & 8.64 & \\
\hline$\% 7$ years or less & 1.92 & & 2.05 & \\
\hline \% No schooling & 0.48 & & 0.82 & \\
\hline Mean age of father in years & 36.8 & 5.64 & 37.12 & 5.49 \\
\hline \multicolumn{5}{|l|}{ Paternal education } \\
\hline$\% 12$ years or more & 89.7 & & 91.52 & \\
\hline$\% 10$ years or less & 6.7 & & 7.14 & \\
\hline$\% 7$ years or less & 3.6 & & 0.89 & \\
\hline \% No schooling & 0 & & 0.45 & \\
\hline Birth order & 1.8 & 1.11 & 1.9 & 1.04 \\
\hline$\%$ female & 50.5 & & 38.5 & \\
\hline$\%$ male & 49.5 & & 51.3 & \\
\hline$\%$ sole parent families & 8.7 & & 7.3 & \\
\hline$\%$ two parent families & 88 & & 76.9 & \\
\hline SDQ Conduct problems & 6.9 & 1.8 & 6.8 & 1.6 \\
\hline SDO Hyperactivity & 8.4 & 2.32 & 8.4 & 2.34 \\
\hline SDQ Anxiety & 6.5 & 1.6 & 6.6 & 1.6 \\
\hline SDO Peer relationships & 6.44 & 1.5 & 6.72 & 1.5 \\
\hline SDO Pro-social behaviours & 12.5 & 1.73 & 12.4 & 1.7 \\
\hline APQ Parental involvement & 39.9 & 4.5 & 39.6 & 5 \\
\hline APQ Parental supervision & 15.54 & 4.01 & 15.06 & 3.9 \\
\hline APQ Positive parenting techniques & 26.26 & 2.37 & 26 & 2.64 \\
\hline APO Parental inconsistent discipline & 13.74 & 3.17 & 13.29 & 3.25 \\
\hline APQ Parents use of corporal punishment & 5.36 & 1.54 & 5.56 & 1.52 \\
\hline
\end{tabular}

\section{Teacher Engagement in the School Component of the Early Intervention Program}

To measure participating teachers' levels of engagement, teachers completed the program implementation subscale of the TSR at post-intervention. All participants selected the same category ( $80 \%$ to $100 \%$ ) on six of the seven items. Overall percentages are presented as follows: in the implementation and facilitation of the EI program, all teachers consistently applied strategies of management, included all students in the curriculum activities, encouraged children to consider pro-social responses, delivered the EI program, encouraged children to participate in the curriculum, and 
adhered to the curriculum delivery guidelines within the range as indicated by the highest end of the 5-point scale showing $80 \%$ to $100 \%$ consistency. Finally, $89 \%$ of teachers maintained contact with parents, where necessary, within the range of $80 \%$ to $100 \%$ consistency.

\section{Behaviour Consultant Engagement in the Early Intervention Program}

The behaviour consultant selected the same category of response ( $80 \%$ to $100 \%)$ for all items on the BCRF. On the program implementation subscale the consultant reported high levels of engagement. Overall percentages measuring the consultant's engagement are presented as follows: the consultant met with indicated children on a weekly basis, employed strategies that closely aligned with the EI program, encouraged indicated children to consider pro-social responses, referred to concepts explored in the program curriculum, made regular weekly contact with each child's teacher, and endeavoured to keep regular communication with each child's parent within the range of $80 \%$ to $100 \%$.

\section{Parent Engagement in Parent Training}

Seventy-two parents (out of 212: 34\%) of the entire EI cohort completed at least one session of training. Of these, 35 (out of 146: 23.9\%) were parents of non-indicated children and 37 (out of 66: 56\%) were parents of indicated children. Of the 35 parents $(31 \%)$ of non-indicated children, 16 participated in one session of the group training, 4 parents attended two sessions and 15 parents participated in three sessions. Of the 37 indicated parents, 11 parents participated in one session of the group-facilitated parent program, 4 parents in two sessions and 14 parents in three sessions. The remaining parents (8) were invited to participate in a 1-hour, one-onone training session with a parent trainer at the child's school or the parent's home. Reported percentages would indicate that participation rates for indicated parents were impressively high for school-based prevention studies. However, it must be noted that very few attended all sessions; many of the parents either attended only one session of the group training or failed to attend any sessions and so were given remedial sessions that gave only a brief introduction to the parenting component of the EI intervention.

There was a significant difference found between the cohort of parents who attended parent training and those who did not attend on the variable of the fathers' levels of education, $F(1,189)=5.30, p<.05$. Fathers who attended parent training indicated higher levels of educational attainment. There were no other differences on subscales of the SDQ (Parent) and SDQ (Teacher) between the children whose parents engaged in parent training and those who did not.

\section{Parent Engagement in the Home Component of the Early Intervention Program}

The PSR was administered at post-intervention to all parents in the EI cohort who completed at least one session of parent training. A Cronbach $\alpha$ level (SPSS) of .85 was calculated on this measure, indicating acceptable internal consistency. Forty-five parents completed the PSR with results indicating high levels of engagement. Eightyfour per cent of parents either agreed or strongly agreed that they felt confident using the strategies learnt $(M=1.98, S D=0.79)$. Seventy-six per cent of parents reported they felt more confident about disciplining their child $(M=2.2, S D=0.79)$. Seventythree per cent of parents indicated that they found the strategies they learnt helped 


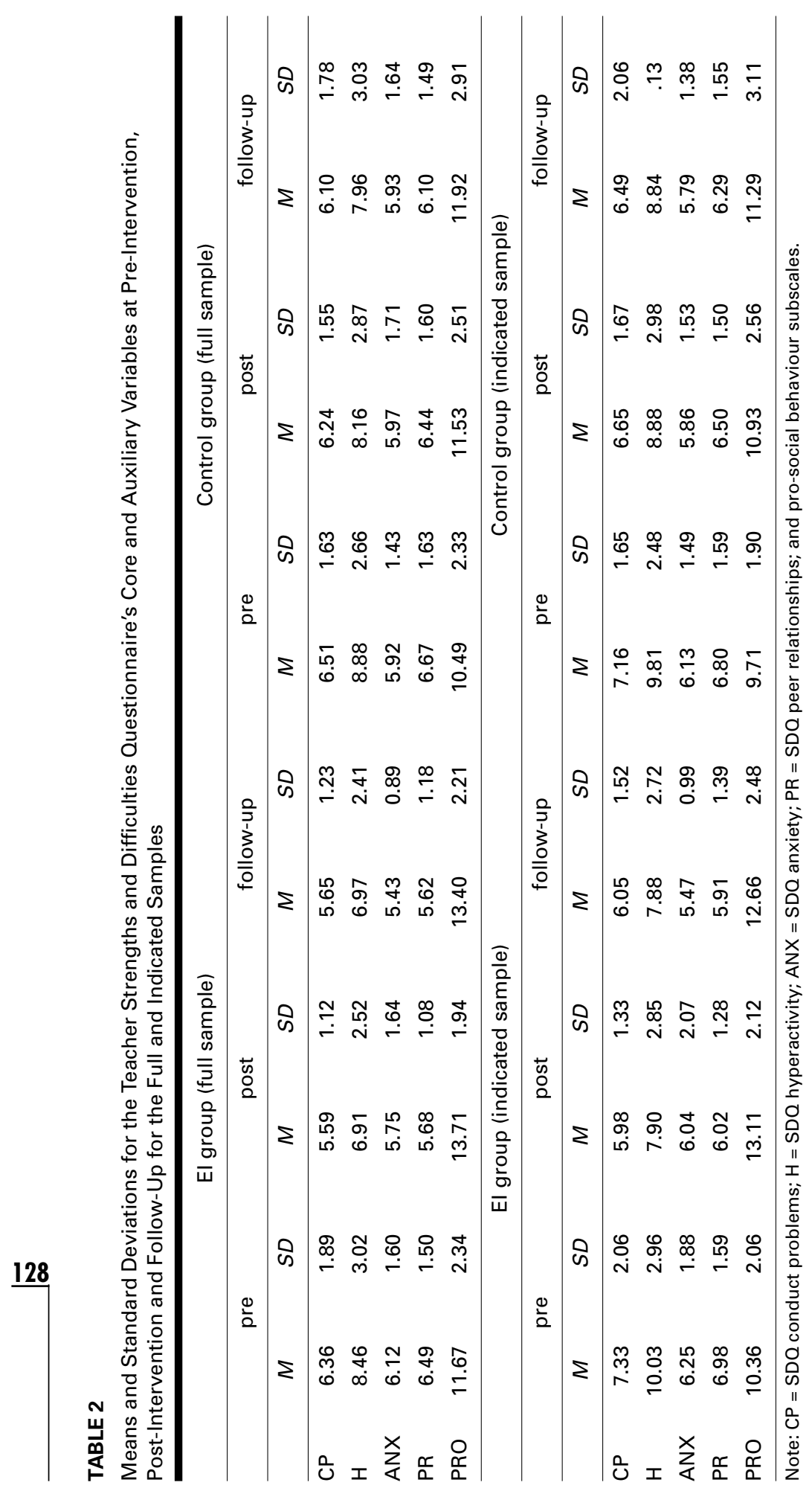


them in disciplining their child $(M=2.20, S D=0.69)$. Sixty-two per cent of parents reported that their ability to manage their child had improved $(M=2.42, S D=$ 0.84). Finally, $93 \%$ of parents indicated that they had kept in touch with their child's teacher throughout the facilitation of the program $(M=1.84, S D=0.67)$.

\section{Service Satisfaction}

Teacher and parent participant satisfaction was measured using overall percentages taken from items on the TSR and PSR respectively at post-intervention. Sixty-two per cent of teachers strongly agreed and $37.5 \%$ agreed that the design of the EI program provided a sound framework for working with children exhibiting problem behaviour $(M=1.38, S D=0.52)$. That the program provided further assistance for children at risk, $87.5 \%$ of teachers strongly agreed and $12.5 \%$ agreed $(M=1.13$, $S D=0.35)$. The percentage of teachers that indicated that as a result of the EI program children were well behaved for $80 \%$ to $100 \%$ of the time was $62.5 \%$, while $37.5 \%$ reported that children were well behaved between $60 \%$ and $79 \%$ of the time $(M=1.38, S D=0.52)$. Seventy-five per cent of teachers indicated that their ability to manage the class group was excellent, while $25 \%$ described their classroom management ability to be good $(M=1.25, S D=0.46)$. Finally, $50 \%$ of the EI teachers reported that indicated children were well behaved for $80 \%$ to $100 \%$ of the time $(M=1.63, S D=0.74), 37.5 \%$ reported that indicated children were well behaved for $60 \%$ to $79 \%$ of the time, and $12.5 \%$ identified that indicated children exhibited pro-social behaviours for $40 \%$ to $59 \%$ of the time. These findings indicate high levels of teacher satisfaction with the EI program.

Of the 45 parents who completed some parent training, $11.1 \%$ strongly agreed and $62.2 \%$ agreed that strategies acquired assisted in the discipline of their child (M $=2.20, S D=0.69)$. The percentage of parents that reported that their ability to manage their child's behaviour had improved was $62.2 \%(M=2.42, S D=0.84)$.

\section{Intervention Participation and Effects}

Given that measures on child behaviour and teaching and parent management were taken from participants involved in the study over time and correlated, repeated measures multivariate analyses of variance (MANOVAs) were therefore used. Where significant time differences, group and interaction effects were found, Bonferroni tests were used to locate the source of the interactions at the .05 level.

\section{Child Behaviour - School Level}

Two repeated measures MANOVAs were undertaken with the between-participants factor of group (EI and control group) and within-participants factor of time (preintervention, post-intervention, and follow-up). These were conducted for the full sample on the SDQ core measures of conduct problem (CP) and hyperactivity $(\mathrm{H})$ and the auxiliary measures of anxiety (ANX), peer relationships (PR), and pro-social (PRO). Table 2 shows means and standard deviations. The MANOVA showed main effects for group, $F(2,236)=4.28, p<.05$; time, $F(4,234)=16.07, p<.01$; and the interaction, $F(4,234)=2.48, p<.05$. Follow-up univariate analyses showed the interaction was significant for both $\mathrm{CP}$ and $\mathrm{H}$. Bonferonni tests were used to locate the source of the interactions. As can be seen from Table 2, the two groups were not different for the $\mathrm{CP}$ and $\mathrm{H}$ subscales at pre, and diverged at post and were significantly different, with the EI group showing lower levels of CP and H. At follow-up 
the EI group appears to maintain its relative gains; however, the difference was no longer significant.

For the measures of ANX, PR, and PRO, the MANOVA revealed main effects for group, $F(3,226)=11.88, p<.01$; time, $F(6,223)=23.80, p<.01$; and the interaction, $F(6,223)=5.94, p<.01$. Follow-up univariate analyses showed the interaction was significant for ANX, PR, and PRO. Bonferroni tests showed that the two groups for the PR subscale were not different at pre, diverged at post and were significantly different, and appeared different at follow-up but were not significant. For the PRO subscale, the two groups were not different at pre, diverged at post and were significantly different, and continued to be significantly different at follow-up. For the ANX subscale, the two groups were not different at pre, diverged at post but were not significantly different, and then reached a level of significant difference in scores at follow-up.

The same MANOVAS were repeated using the smaller indicated sample with the between-participants factor of group (EI and control group) and within-participants factor of time (pre-intervention, post-intervention, and follow-up) on the same core variables. Table 2 shows means and standard deviations. Results were first analysed on dependent measures of $\mathrm{CP}$ and $\mathrm{H}$ using a 2 (group: EI versus control) $\times$ 3 (time: pre, post, fu) MANOVA. The MANOVA showed no main effects for group, $F(2,134)=1.11, p>.05$; a main effect for time, $F(4,132)=14.49, p<.01$; and an interaction, $F(4,132)=2.72, p<.05$. Follow-up univariate analyses showed the interaction was significant for both $\mathrm{CP}$ and $\mathrm{H}$. Results from Table 2 show the two groups were not different for the $\mathrm{CP}$ and $\mathrm{H}$ subscales at pre, diverged at post, and remained different at follow-up; however, the Bonferonni tests failed to find significant differences at either point.

For the measures of ANX, PR, and PRO, the MANOVA revealed main effects for group, $F(3,125)=5.72, p<.01$; time, $F(6,122)=18.56$, $p<.01$; and the interaction, $F(6,122)=3.87, p<.01$. Follow-up univariate analyses showed the interaction was significant for PR and PRO. Table 2 indicates that the two groups for the PRO subscale were not different at pre, diverged at post and were significantly different, and remained different at follow-up but no longer had significantly different scores. The two groups for the PR subscales were not different at pre, diverged at post but were not significantly different, and remained not significant at follow-up.

\section{Parenting Practices}

Two repeated measures MANOVAs with the between-participants factor of group (EI and control group) and within-participants factor of time (pre-intervention, post-intervention, and follow-up) were conducted on each subscale of the APQ for the entire cohort of participants. Table 3 shows means and standard deviations at pre, post, and follow-up. The MANOVA showed no main effects for group, $F(5$, $224)=2.09, p>.05$; main effects for time, $F(10,219)=4.97, p<.01$; and no interaction, $F(10,219)=1.45, p>.05$. On each variable the time effects reflected small but consistent decreases on measures over time.

The same MANOVAs were repeated using the sample whose parents had received the intervention treatment and the full control sample, with the betweenparticipants factor of group (EI - treatment sample, and control group) and within-participants factor of time (pre-intervention, post-intervention, and followup) on each subscale of the APQ. Table 3 shows means and standard deviations at pre, post, and follow-up. The MANOVA for the five variables showed no main 


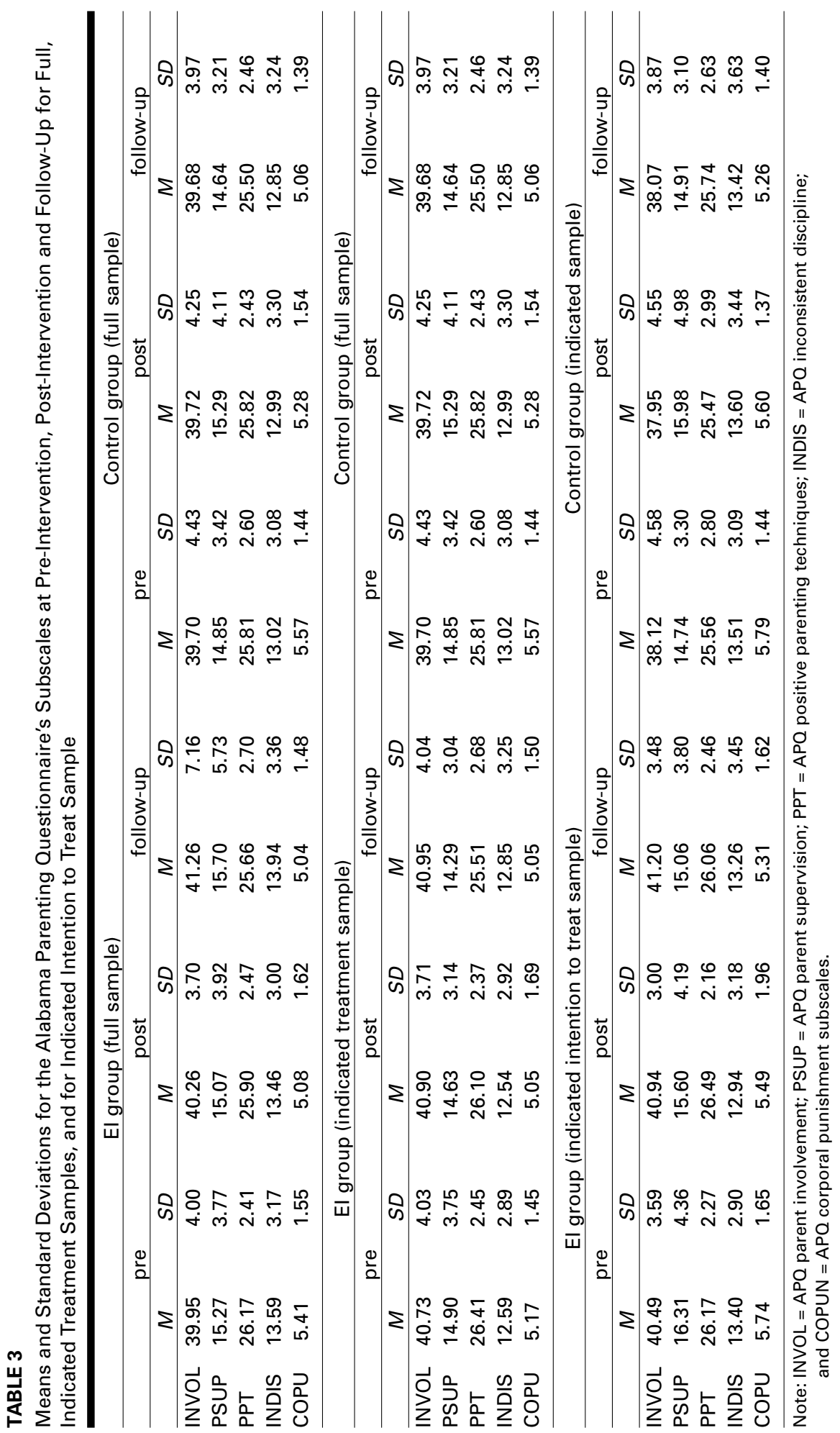




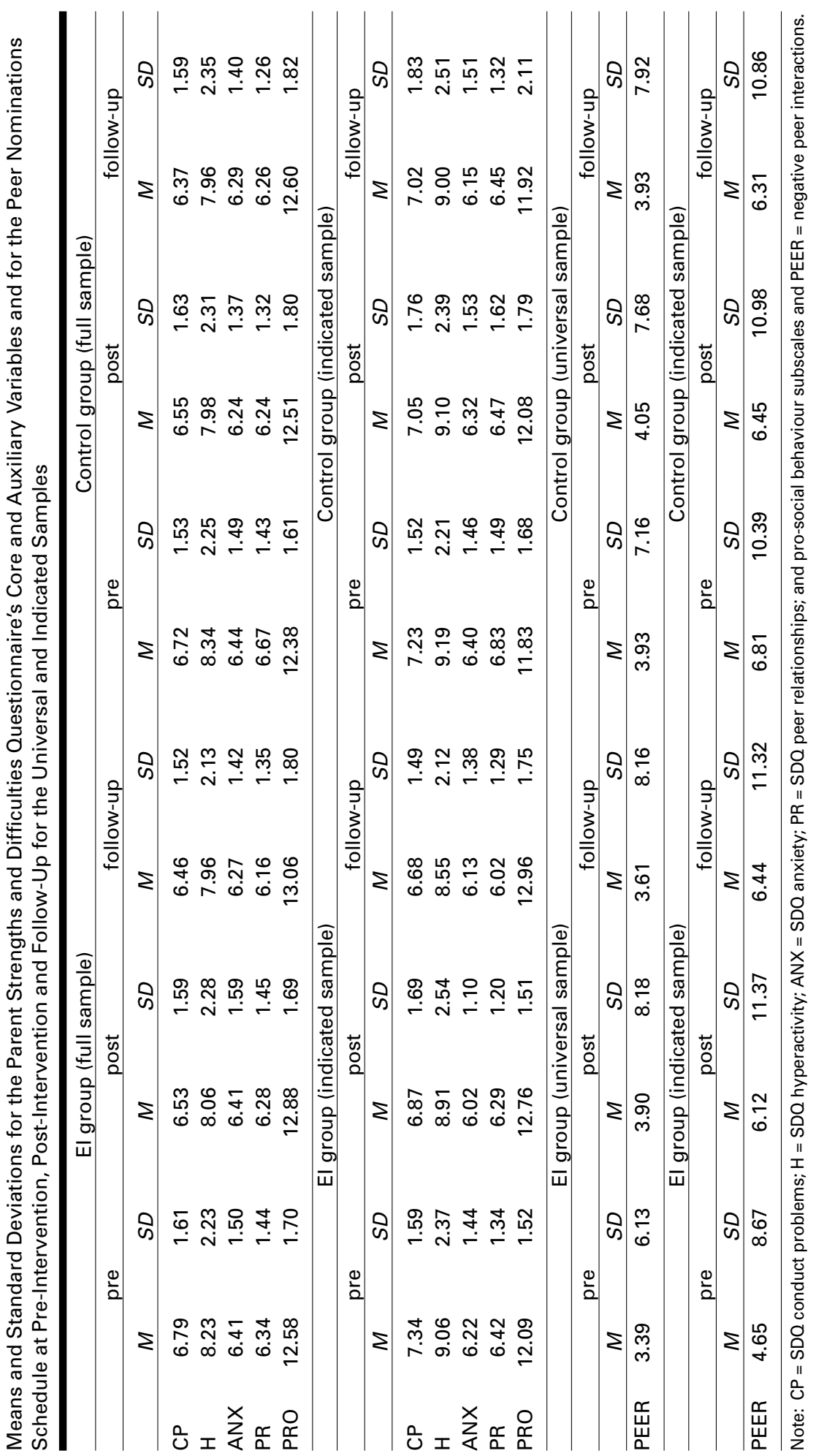


effects for group, $F(5,157)=.69, p>.05$; main effects for time, $F(10,152)=2.61$, $p<.01$; and no interaction, $F(10,152)=.64, p>.05$. Again, on each variable the time effects reflected small but consistent decreases on measures over time.

Two repeated measures MANOVAs were also conducted on each subscale of the APQ for the indicated sample with the between-participants factor of group (EI intention to treat, and control group - indicated) and within-participants factor of time (pre-intervention, post-intervention, and follow-up). Table 3 shows means and standard deviations at pre, post, and follow-up. The MANOVA showed main effects for group, $F(5,72)=3.96$, $p<.01$; no main effects for time, $F(10,67)=1.55$, $p>.05$; and no interaction, $F(10,67)=0.97, p>.05$. On each variable the group effects reflected small but consistent decreases on measures over time.

\section{Child Behaviour - Home Level}

Two repeated measures MANOVAs with the between-participants factor of group (EI and control group) and within-participants factor of time (pre-intervention, postintervention, and follow-up) for the full sample were conducted on each subscale of the parent version of the SDQ. Table 4 shows means and standard deviations at pre, post, and follow-up. The MANOVA for core variables showed no main effects for group, $F(2,349)=0.09, p>.05$; main effects for time, $F(4,347)=6.71, p<.01$; and no interaction, $F(4,347)=.60, p>.05$. For the auxiliary variables the MANOVA showed no main effects for group, $F(3,330)=1.93$, $p>.01$; main effects for time, $F(6,327)=4.66, p<.01$; and no interaction, $F(6,327)=1.60, p>.05$. On all measures the time effects reflected small but consistent decreases on measures over time.

The same MANOVA was repeated using the indicated sample with the between-participants factor of group (EI and control group) and within-participants factor of time (pre-intervention, post-intervention, and follow-up) on each subscale of the parent version of the SDQ. Table 4 shows means and standard deviations at pre, post, and follow-up. The MANOVA showed no main effects for group, $F(2,106)=0.21, p>.05$; main effects for time, $F(4,104)=2.82, p<.05$; and no interaction, $F(4,104)=0.74, p>.05$. Results were then analysed on auxiliary variables with the MANOVA showing no main effects for group, $F(3,94)=$ $1.88, p>.05$; main effects for time, $F(6,91)=2.85, p<.01$; and no interaction, $F(6,91)=1.52, p>.05$. Again, on all measures the time effects reflected small but consistent decreases on measures over time.

\section{Peer Relationships}

Two repeated measures MANOVAs with the between-participants factor of group (EI and control group) and within-participants factor of time (pre-intervention, post-intervention, and follow-up) for the entire cohort of participants were conducted on the variables of the PNS. Table 4 shows means and standard deviations for the variables' scores at pre, post, and follow-up. The MANOVA showed no main effects for time, $F(2,506)=0.82, p>.05$, and no interaction, $F(2,506)=$ $0.29, p>.05$. The same MANOVAs were repeated using the indicated sample with the between-participants factor of group (EI and control group) and within-participants factor of time (pre-intervention, post-intervention, and follow-up) on the variables of the PSN. The MANOVA showed no main effects for time, $F(2,174)=$ $.58, p>.05$, and no interaction, $F(2,174)=1.65, p>.05$. 


\section{Diagnostic Data}

Table 5 shows the follow-up clinical status of the indicated participants in the study. At the 6-month follow-up period chi-square analyses showed there were no significant differences between the EI and control group for any diagnosis, $\chi^{2}(1, N=136)$ $=1.10, p=.213$; internalising diagnosis, $\chi^{2}(1, N=134)=3.77, p=.063$; and externalising diagnosis, $\chi^{2}(1, N=134)=0.094, p=.519$. ANOVA was used to test the significance of differences between mean scores for the highest severity rating of diagnosis at 6-months follow-up. The mean and standard deviation scores for the EI group on the highest rating of severity were $M=0.77$ and $S D=1.43$. For the control group the mean and standard deviation scores for the highest severity rating were $M=0.45$ and $S D=0.99$. However, there were no statistically significant differences for group $F(1,132)=2.38, p>.05$ at the 6 -month follow-up period.

\section{Discussion}

The main aim of this study was to evaluate the efficacy of the EI program. The results at the school level were promising. The rate of children recruited into the study was high and teachers and the behaviour consultant were actively engaged throughout the implementation period of the program. At the school level an intervention effect was found at post-intervention with significant differences between the EI full cohort compared with the full control group. The findings of the SDQ (Teacher) reports indicated changes in the behaviour of children in the intervention group at post-intervention with some evidence of sustained changes at 6 month follow-up.

The home component of the EI program revealed no significant effects at postintervention or 6-month follow-up. Based on current advances in preventative research, the EI home component should serve to reduce problem behaviour. The most likely explanation for the lack of significant change could be that the majority of parents of indicated children attended only one third of the parent-training component of the EI intervention. This reduced dosage may have accounted for a lack of reported change in parenting practices, thus potentially influencing the degree of behaviour change in the child. Despite these findings, similar studies have reported lower incidences in problem behaviours at the home level. Such outcomes support the significance of other intervention frameworks in reducing problem behaviours in children in the home setting. Programs such as Head Start (Webster-Stratton, 1998), First Step to Success (Walker et al., 1998) and Fast Track (Conduct Problems Prevention Research Group, 2002) support the influences of early intervention models on the family domain. Despite parallels between the methodologies of the EI trial and other interventions, we failed to find evidence of positive outcomes at the home level for the EI program. As suggested, limited engagement in the parent-training component for parents of indicated children would most likely account for the lack of behaviour change. Participation rates for parents involved in the Head Start, First Step to Success and Fast Track trials were high, and were instrumental in the facilitation of significant outcomes. In the aforementioned trials, high levels of engagement were encouraged through the provision of incentives such as regular payment to parents for their involvement. While such incentives assist in recruitment and retention, financial constraints associated with the present study did not allow for this approach to be adopted. Further, there is some 
TABLE 5

Diagnostic Data at 6-Month Follow-Up for the Indicated Sample

\begin{tabular}{lcc}
\hline & El Group $(n=60) \%$ & Control Group $(n=76) \%$ \\
\hline Children with: & $16.7 \%$ & \\
Any diagnosis & $8.3 \%$ & $10.5 \%$ \\
Internalising diagnosis & $6.7 \%$ & $1.4 \%$ \\
Externalising diagnosis & $5 \%$ & $5.4 \%$ \\
Attention-deficit/hyperactivity disorder & $6.7 \%$ & $2.7 \%$ \\
Enuresis & $1.7 \%$ & $5.3 \%$ \\
Separation anxiety disorder & $3.3 \%$ & $2.7 \%$ \\
Oppositional defiant disorder & $1.7 \%$ & $6.7 \%$ \\
Social phobia & $8.3 \%$ & $2.7 \%$ \\
Specific phobia & $1.7 \%$ & $6.7 \%$ \\
Adjustment disorder & $0 \%$ & $4 \%$ \\
Encopresis & $3.3 \%$ & $4 \%$ \\
School refusal & $1.7 \%$ & $0 \%$ \\
Conduct disorder & $1.7 \%$ & $1 \%$ \\
Major depressive disorder & $0 \%$ & $0 \%$ \\
Depressive disorder & $1.3 \%$ \\
\hline
\end{tabular}

question concerning the sustainability of such an approach for regular schools and community groups.

In terms of the social validity of the EI program, teachers, parents and the behaviour consultant reported high levels of satisfaction with the program. The high rates of participation, retention and satisfaction give weight to this finding. Specifically, teacher, parent and consultant participants reported increased confidence in managing problem behaviours in children. Further, teachers indicated that they had benefited from the utility of the program design. Parent engagement in the program was high for prevention studies with $30.5 \%$ of parents participating. However, for parents of children in the indicated cohort participation rates were low and as discussed in an earlier section may have accounted for the lack of behaviour change. Despite these lower attendance rates, the full sample generally reported high levels of satisfaction with the program. Retention rates throughout the evaluation were also high. The attrition rate from pre-to post-intervention was $99 \%$, and $96 \%$ from pre-intervention to follow-up, indicating parental support for children's involvement.

\section{Strengths and Limitations}

There were a number of strengths in the study. First, the sample size for the investigation was large and the retention rate high. Second, the sociodemographic composition of participating schools was homogenous, allowing for valid comparisons between school groups. Third, teachers, administrative staff and parents involved in the trial were supportive of the research, facilitating a dynamic conducive to program implementation and data collection. Fourth, participating teachers were consistent in the facilitation of the EI program, ensuring high treatment integrity. 
Clearly, the strengths of this trial lend support to community-based early intervention and prevention programs that offer assistance to schools and families in remediating the effects of problem behaviour in children.

There were a number of limitations of the study that were largely influenced by time and resource restraints that may restrict the interpretation of findings. First, teachers were responsible for screening of children for inclusion in the trial, as well as completing outcome and treatment integrity measures. Using additional measures to supplement the teacher's evaluation may have given weight to findings at the school level. Second, this study was limited to follow-up data at the 6-month follow-up period. To understand the effects of the EI program over time, longer-term follow-up (e.g., 2 years) is necessary. Third, the emphasis of the school component on teacher management and social skills development may fail to adequately account for other psychosocial variables such as academic dysfunction (Coie et al., 1993). Fourth, there were a number of difficulties in encouraging parental involvement in the EI intervention. Clearly, alternative approaches to engage more at-risk parents are necessary to enhance treatment outcomes.

Based on the present findings, a number of recommendations can be made that serve to direct future research. First, research in prevention should investigate alternative methods for successfully engaging at-risk families into school-based programs. Second, the current study shows that regular teachers are capable of implementing programs and further studies should give consideration to building on this with an emphasis on effectiveness trials using real-world staff. Finally, future research initiatives may want to consider the significance of home-school partnerships to help engage parents of problem children and foster generalisation between any positive change in the school and home.

\section{References}

Achenbach, T.M., \& Edelbrock, C.S. (1991). Manual for the Child Behavior Checklist and Revised Child Behavior Profile. Burlington, VT: University of Vermont Department of Psychiatry.

American Psychiatric Association. (1994). Diagnostic and statistical manual of mental disorders (4th ed.). Washington, DC: Author.

American Psychiatric Association. (2000). Diagnostic and statistical manual of mental disorders (4th ed., text Rev.). Washington, DC: Author.

August, G.J., Realmuto, G.M., Hektner, J.M., \& Bloomquist, M.L. (2001). An integrated components preventative intervention for aggressive elementary school children: The early risers program. Journal of Consulting and Clinical Psychology, 69(4), 614-626.

Coie, J.D., Watt, N.F., West, S.G., Hawkins, J.D., Asarnow, J.R., Markman, H.J., et al. (1993). The science of prevention: A conceptual framework and some directions for a national research program. American Psychologist, 48(10), 1013-1022.

The Conduct Problems Prevention Research Group. (2002). Evaluation of the first 3 years of the fast track prevention trial with children at high risk for adolescent conduct problems. Journal of Abnormal Child Psychology, 30(1), 19-35.

Crick, N.R., \& Grotpeter, J.K. (1995). Relational aggression, gender, and social-psychological adjustment. Child Development, 66(3), 710-722.

Dadds, M.R., Maujean, A., \& Fraser, J.A. (2003). Parenting and conduct problems in children: Australian data and psychometric properties of the Alabama parenting questionnaire. Australian Psychologist, 38(3), 238-241.

Dadds, M., Spence, S.H., Holland, D.E., Barrett, P.M., \& Laurens, K.R. (1997). Prevention and early intervention for anxiety disorders: A controlled trial. Journal of Consulting and Clinical Psychology, 65(4), 627-635. 
Goodman, R. (2001). Psychometric properties of the strengths and difficulties questionnaire. Journal of the American Academy of Child EB Adolescent Psychiatry, 40(11), 1337-1345.

Goodman, R. (1997). The Strengths and Difficulties Questionnaire: A research note. Journal of Child Psychology and Psychiatry, 38, 581-586.

Hawes, D.J., \& Dadds, M.R. (2004). Australian data and psychometric properties for the strengths and difficulties questionnaire. Australian and New Zealand Journal of Psychiatry, 38, 644-651.

Holland, D., \& Dadds, M.R. (1997). The Diagnostic Interview Schedule for Children, Adolescents, and Parents. Brisbane, Australia: Griffith University.

Johnson, S., Barrett, P.M., Dadds, M.R., Fox, T., \& Shortt, A. (1999). The diagnostic interview schedule for children, adolescents, and parents: Initial reliability and validity data. Behaviour Change, 16(3) 155-164.

Kim-Cohen, J., Caspi, A., Moffitt, T.E., Harrington, H., Milne, B.J., \& Poulten, R. (2003). Prior juvenile diagnoses in adults with mental disorders. Archives of General Psychiatry, 60, 709-717.

Larmar, S.A. (2002a). Encouraging Positive Behaviour in the Classroom. Unpublished manual.

Larmar, S.A. (2002b). Encouraging Positive Behaviour in Young Children. Unpublished manual.

Larmar, S.A. (2002c). The Early Impact Program: A Program for Encouraging Positive Behaviour in Young Children. Unpublished manual.

Larmar, S.A., \& Dadds, M.R. (2002a). Behaviour Consultant Report Form. Unpublished measure.

Larmar, S.A., \& Dadds, M.R. (2002b). Parent Self-Report. Unpublished measure.

Larmar, S.A., \& Dadds, M.R. (2002c). Peer Nomination Interview Schedule. Unpublished measure.

Larmar, S.A., \& Dadds, M.R. (2002d). Teacher Self-Report. Unpublished measure.

Patterson, G.R., DeGarmo, D.S., \& Knutson, N. (2000). Hyperactive and antisocial behavior: Comorbid or two points in the same process. Development and Psychopathology, 12, 91-106.

Sanders, M.R., Tully, L.A., Baade, P.D., Lynch, M.E., Heywood, A.H., Pollard, G.E., et al. (1999). A survey of parenting practices in Queensland: Implications for mental health promotion. Health Promotion Journal of Australia, 9(2), 105-114.

Shelton, K.K., Frick, P.J., \& Wooton, J. (1996). Assessment of parenting practices in families of elementary school-age children. Journal of Clinical Child Psychology, 25, 317-329.

Walker, H.M., Severson, H.H., Feil, E.G., Stiller, B., \& Golly, A. (1998). First step to success: Intervening at the point of school entry to prevent antisocial behavior patterns. Psychology in the Schools, 35(3), 259-269.

Webster-Stratton, C. (1998). Preventing conduct problems in head start children: Strengthening parenting competencies. Journal of Consulting and Clinical Psychology, 66(5), 715-730. 